Cuadernos de Gobierno y Administración Pública ISSN: e-2341-4839

http://dx.doi.org/10.5209/CGAP.60609

\title{
La promoción de la cultura de defensa en las universidades españolas: Evaluación de diseño del Programa de Colaboración del Ministerio de Defensa
}

\author{
Alberto Bueno*
}

Recibido: 16/03/2018 / Aceptado: 12/05/2018

Resumen. Este artículo presenta una evaluación de diseño del Programa de Colaboración con las Universidades del Ministerio de Defensa, por ser el instrumento de planificación e implementación de la política pública de promoción de la cultura de defensa en ese ámbito. Desde el enfoque del análisis de políticas públicas, valora su racionalidad, pertinencia, coherencia interna y coherencia externa mediante diversas técnicas analíticas. Con esta investigación se pretende, por un lado, profundizar en el conocimiento de esta política y evaluar la alternativa formulada y, por otro, ofrecer una serie de recomendaciones de política, las cuales podrían ser de utilidad para los decisores públicos en la elaboración del nuevo Plan Director de Cultura de Defensa.

Palabras clave: políticas públicas, evaluación, cultura de defensa, universidad, y política de defensa.

\section{The promotion of the culture of defence in spanish universities: Design evaluation of the collaboration Program of the Ministry of Defence}

\begin{abstract}
This article presents a design evaluation of the Collaboration with Universities Program of the Spanish Ministry of Defence, which represents the planning and implementation instrument for the public policy to promote the culture of defence in that field. From the perspective of the policy analysis, it assesses its rationality, relevance, internal coherence and external coherence through various analytical techniques. On the one hand, this research is intended to deepen the knowledge of this policy and to evaluate the formulated alternative and, on the other hand, to offer a series of policy recommendations which could be useful for public decision-makers in the preparation of the new Culture of Defence Director Plan.
\end{abstract}

Keywords: Policy Analysis, Policy Evaluation, Culture of Defence, University, Spain Defence Policy.

Sumario. 1. Introducción. 2. Enfoque teórico y metodológico. 3. Evaluación de diseño del Programa de Colaboración con las Universidades. 3.1. Un contexto de recelos e intereses mutuos. 3.2. El PCU como instrumento de actuación pública: objetivos y acciones. 3.3. Resultados de investigación sobre de la racionalidad, pertinencia y coherencia interna y externa del programa. 4. Conclusiones. 5. Recomendaciones de política. Documentos. Bibliografía.

Cómo citar: Bueno A., «La promoción de la cultura de defensa en las universidades españolas: Evaluación de diseño del Programa de Colaboración del Ministerio de Defensa», en Cuadernos de Gobierno y Administración Pública 5-1, 27-47.

\footnotetext{
* Universidad de Granada. Esta investigación se ha beneficiado del programa de ayudas para contratos predoctorales de Formación de Profesorado Universitario 2015 del Ministerio de Educación, Cultura y Deporte. Asimismo, el autor agradece los comentarios de los evaluadores anónimos, los cuales han mejorado, sin duda, el manuscrito original.
} 


\section{Introducción}

Uno de los ejes más importantes de la política de defensa española en las últimas dos décadas ha sido el fomento de la llamada cultura de defensa ${ }^{1}$. La relevancia de esta política pública queda reflejada en su inclusión en los principales documentos jurídico-políticos de defensa y seguridad promulgados en España desde la década del 2000: el Libro Blanco de la Defensa del año 2000, las directivas de defensa nacional desde esa misma fecha (2000 [directiva que formula por primera vez esta política], 2004, 2008 y 2012), la Revisión Estratégica de la Defensa de 2003, la Ley de Defensa Nacional de 2005, las estrategias de seguridad nacional publicadas en los años 2011 y 2013 respectivamente, y la Ley de Seguridad Nacional de 2015. Además, ha sido un componente del proceso de transformación militar español (Colom, 2011).

Para definir y conformar el proceso de implementación de esta política, entre los años 2002 y 2003 se publicaron el Plan Director de Cultura de Defensa (en adelante, PDCD) y sus respectivos planes derivados, dedicado cada uno a sectores concretos de actuación: Plan de Comunicación Social, Plan de Colaboración con el Sistema Educativo, Plan Sociedad - Fuerzas Armadas, Plan de Colaboración de las Administraciones Públicas, Plan de Acción Cultural y Plan de Investigación. Desde esa fecha, permanecen en vigor y, por ende, están todavía en aplicación.

Esta iniciativa de intervención pública fijó su atención en las universidades y en la población de analistas e investigadores, entonces en ciernes, del área de estudios de seguridad internacional y defensa. El motivo era doble: por un lado, por ser actores clave para difundir el conocimiento y la concienciación sobre la importancia de tales políticas; por otro, para nutrir un músculo científico que, en aquel momento, se entendía débil dado el escaso número tanto de think-tanks como de asignaturas relacionadas en los programas de estudio universitarios. Por estas razones, dentro del Plan derivado de Colaboración con el Sistema Educativo (PCSE, en adelante), se elaboró específicamente un Programa de Colaboración con las Universidades (PCU), cuyo objetivo entre otros es "promover el estudio, debate e investigación [...] sobre las cuestiones relacionadas con la paz, la seguridad y la defensa". Y relacionado con los mismos objetivos, el Plan derivado de Investigación y sus programas correspondientes.

La evolución de esta política pública ha justificado su análisis en diversas obras, destacando entre ellas: el "Documento de Trabajo" de Marrero Rocha (2007) para la Fundación Alternativas; sendos monográficos de las Universidades de Granada (Trujillo y García Sistac, 2006) y Córdoba (López Mora y Ballesteros, 2011), respectivamente, en colaboración con organismos de la Defensa; un "Curso Internacional de Defensa" del Ministerio de Defensa (AA.VV., 2014); dos "Cuadernos de Estrategia" del Instituto Español de Estudios Estratégicos (2011, 2015); así como dos números monográficos de la revista ARBOR (Ballesteros y Fernández Vargas, 2008; Fernández Vargas y Rodríguez-Toubes, 2014). Asimismo, ha sido objeto de atención habitual en documentos de centros de análisis o prensa especializada.

También denominada como cultura de seguridad y defensa, cultura de seguridad, o cultura de seguridad nacional. Esta panoplia de distintas voces ilustra la adaptación terminológica a una realidad en constante transformación en el campo de la seguridad y la defensa. 
Esta prolífica producción muestra la relevancia de esta política pública. En general, dichos textos han incidido en su conceptualización o caracterización, o en la explicación y difusión de muchas de las acciones y actividades llevadas a cabo. El enfoque divulgativo, antes que científico, de la mayoría de estos trabajos es la nota predominante.

Son numerosos los artículos que explican las relaciones entre las universidades españolas y las Fuerzas Armadas (FAS), pero se adolece de investigaciones en profundidad sobre su proceso de desarrollo y efectos. Se observa un claro déficit en cuanto a su estudio desde la disciplina de la Ciencia Política y de la Administración y, en particular, desde el campo del análisis y la evaluación de políticas públicas. Dicha circunstancia coincide, no obstante, con una escasa producción científica en general acerca de la política de defensa española (Jordán, 2017). Sólo los trabajos de Marrero Rocha (2011) y García Palomero (2014), sin ser investigaciones de evaluación sensu stricto ni enmarcarse explícitamente en el terreno de la politología, ofrecen algunos datos sobre la promoción de la cultura de defensa en el ámbito universitario y sus resultados ${ }^{2}$. Ambos trabajos, junto con el análisis de los artículos publicados en revistas españolas especializadas o los grupos de trabajos sobre seguridad y defensa conformados para los congresos bianuales de la Asociación Española de Ciencia Política y de la Administración (Bueno, 2017), permiten apreciar, pese a todo, el progreso de este tema en las aulas universitarias españolas.

Como corolario, ante la ausencia de este tipo de investigaciones sobre estos planes y programas de cultura de defensa, se considera de utilidad evaluar los instrumentos fundamentales de su programación, los cuales han configurado todo el desarrollo de esta política pública. Con esta finalidad, en este artículo se aplica una evaluación de diseño a los elementos constitutivos del PCU. De tal forma, el objetivo que se persigue es doble: por un lado, valorar su estructura y ordenación lógica, obteniendo información sobre su contenido y profundizando en el conocimiento acerca de la política de promoción de la cultura de defensa; por otro, recomendar diversas mejoras del programa. Éste segundo se establece atendiendo a la publicación en diciembre de 2017 de una nueva Estrategia de Seguridad Nacional ${ }^{3}$ y el propósito de elaborar un nuevo Plan Director de Cultura de Defensa por parte del Ministerio, tal y como adelantó la ministra titular de la cartera, $\mathrm{M}^{\mathrm{a}}$ Dolores de Cospedal, en su comparecencia ante la Comisión de Defensa del Senado en febrero de 2017 (Ministerio de Defensa, 2017: 12).

La estructura del artículo es la siguiente: en primer lugar, se presentan el enfoque teórico y la metodología empleadas. En el epígrafe tercero, se explica cuáles son los rasgos fundamentales de esta política y cuál ha sido su despliegue en el ámbito de la educación superior; a continuación, se expone la evaluación de diseño realizada. Por último, se ofrecen una serie de conclusiones que orientan las recomendaciones de política (policy recommendations) finales.

2 El trabajo de Marrero Rocha, basado parcialmente en la realización de un cuestionario a diversos académicos, señala cómo la política de promoción de la cultura de defensa ha sido una de los factores de consolidación de estos estudios en España. Por su parte, García Palomero se centra en el análisis de tesis doctorales publicadas: una de sus conclusiones es la ausencia de investigaciones de este nivel sobre dichas materias.

3 La Estrategia de Seguridad Nacional de 2017, como la de 2013, desde su enfoque integral, contempla entre sus líneas estratégicas de acción la defensa nacional y con ella el fomento de la cultura de defensa. 


\section{Enfoque teórico y metodológico}

El doble objetivo de este artículo está orientado por la doble perspectiva que fundamenta el análisis de políticas públicas, el cual se dirige hacia los contenidos y los procesos de las políticas, bien para incrementar el conocimiento sobre las mismas, bien para mejorar la toma de decisiones (Hogwood y Gunn, 1984). Estos atributos contribuyen a que pueda presentar un carácter descriptivo, explicativo, prescriptivo o normativo. Esa doble orientación, definida originalmente por Harold D. Lasswell (1970) como el knowledge of (análisis de) las políticas y el knowledge in (análisis para), ha guiado la investigación efectuada, interesada tanto por el conocimiento acerca del desarrollo de la política de promoción de la cultura de defensa como por su perfeccionamiento.

Por su parte, la evaluación de políticas, como integrante del policy analysis, no sólo recoge este enfoque, sino que se sitúa en "la frontera entre el análisis de y el análisis para las políticas" (Pérez Sánchez, 2005: 60). Bajo estas coordenadas, la investigación evaluativa (Briones, 1991; Bartolomé, 1998, Trinidad, 1995) pretende generar conocimiento y mejorar el estado del arte de la política en cuestión (Rossi y Freeman; Rutman, 1984b), así como apoyar el proceso decisional de la intervención pública (Weiss, 1972; Chelimsky, 1987). De tal forma, se liga su uso académico con la investigación social aplicada (Agencia de Evaluación y Calidad [AEVAL], 2010), una lógica que, como se ha mencionado, orienta este trabajo. El análisis y evaluación de políticas públicas, en su afán por buscar la generación de información relevante sobre un determinado problema (Ballart, 1992), se concibe en definitiva como un campo de investigación científica y como un método de trabajo (Mèny y Thoening, 1992).

Bajo este razonamiento, la actividad reflexiva que supone la evaluación (Bartolomé, 1995, en Trinidad, 1995; Nirenberg, 2013; Gutiérrez Díaz, 2014) se caracteriza por la aplicación sistemática y aplicada de las teorías y métodos de investigación propios de las Ciencias Sociales (Anguera y Moscoso, 2008; Ballart, 1992; Fernández-Ballesteros, 1996), de donde deriva su multidisciplinariedad (AEVAL, 2010). La evaluación se sitúa en un continuum entre su construcción "racional" (Weiss, 1987: 47) y su "creatividad" como "proceso conceptual" (Stufflebeam y Shinkfield, 1985: 44-45). Su finalidad es la de obtener datos válidos, fiables, suficientes y relevantes (Aguilar Idáñez y Ander-Egg, 1994) que permitan valorar la acción de la autoridad pública. Éstos son, por tanto, los postulados que presiden esta investigación.

Asimismo, la evaluación realizada se incardina dentro de la corriente académica (Alvira, 1991; Aguilar Idáñez y Ander-Egg, 1994; Cirera y Vélez, 2009; Alemán y Trinidad, 2012; Gutiérrez Díaz, 2014) que concibe la investigación evaluativa, no sólo como el análisis, la interpretación, el conocimiento y el juicio sobre los resultados y efectos, sino también sobre las necesidades que motivan la intervención, su conceptualización y diseño, sus procesos de implementación y los instrumentos empleado.

En particular, estudia y evalúa el diseño del PCU del PCSE, derivado a su vez del PDCD. Dicha investigación sistemática y aplicada revisa los componentes y la forma en que se ha articulado la intervención (Cicera y Vélez, 2000), llevando a cabo un análisis lógico del programa en relación al problema que quiere solucionar: en este caso, el PCU intenta dar respuesta al problema, según entienden los decisores de Defensa, de la escasa cultura de defensa de la comunidad universitaria española. 
Partiendo de la investigación de sus elementos constitutivos (Gutiérrez Díaz, 2014), proporciona información para estimar hasta qué punto el diseño del programa sirve a los objetivos estipulados, además de comprender y explicar en mayor profundidad cómo se desarrollan las intervenciones públicas (GV, n.d.). En definitiva, esta evaluación quiere dar respuesta a la pregunta de si es "la mejor alternativa posible para dar respuesta a las necesidades objeto de la política" (Alemán y Trinidad, 2012: 133).

Para ello, juzga la pertinencia formal y potencial, la racionalidad intrínseca (AEVAL, 2010; GV, n.d.), así como la coherencia interna (Aguilar Idáñez y Ander-Egg, 1994) y externa (Cirera y Vélez, 2000; GV, n.d.) del programa. En general, las dos primeras dimensiones vinculan la naturaleza y características del problema detectado con los objetivos establecidos. La coherencia se refiere al análisis de la jerarquía de objetivos (interna) y a la estrategia seguida en relación con otros programas de intervención (externa).

En particular, cada una de estas dimensiones se centra en distintos aspectos: la racionalidad evalúa la veracidad y calidad del diagnóstico, analizando los problemas descritos y señalando si son explícitos, claros, rigurosos, completos y medibles, al igual que las fuentes de información utilizadas para su enunciación. La pertinencia, por su parte, estudia la calidad de los objetivos del programa con el fin de comprobar si éstos son "válidos para lo que el programa trata de conseguir" (Rutman, 1984a: 29), indicando cómo son definidos (y si admiten interpretaciones o no), si responden a una sola meta, si son claros y concretos, si cada uno se corresponde con un sólo resultado o producto, y que esté previsto algún límite temporal para su consecución. En lo que respecta a la coherencia interna, ésta analiza la lógica de la programación y la jerarquización de los objetivos. Por último, la coherencia externa valora el grado de correspondencia entre el programa en evaluación y otras intervenciones que se están ejecutando en un mismo espacio temporal o físico-territorial.

En cuanto a las características propias de la investigación llevada a cabo, ésta se caracterizada por ser una evaluación externa ${ }^{4}$, proactiva $^{5}$, producida durante la vida del programa ${ }^{6}$, formal y sustancial ${ }^{7}$, y parcial ${ }^{8}$. Respecto al enfoque metodológico, se ha optado por uno cualitativo, siguiendo el consolidado criterio de Amezcua y Jiménez Lara (1996) de aplicar técnicas cualitativas para la evaluación de diseño. El estudio de la información secundaria cualitativa se ha realizado basándose en los documentos jurídico-políticos estratégicos enumerados y aplicables al PCU, así como un estudio bibliográfico para analizar el contexto (Pérez-Llantada y López de la Llave, 1999). Considerando al mismo tiempo el carácter multidisciplinar (Alvira, 1991), abierto y flexible (AEVAL, 2010) de la evaluación, se han seleccionado distintas herramientas técnicas para evaluar las anteriores dimensiones: indicadores y diagrama de problemas para la racionalidad; análisis DAFO para la pertinencia; árbol de

\footnotetext{
Un requisito destacado por Alemán y Trinidad (2012).

Dado que también se exponen una serie de policy recommendations en el epígrafe final.

6 Si bien la literatura recomienda que las evaluaciones de diseño sean ex-ante, los objetivos de investigación fuerzan a elaborarla con este enfoque, asumiendo también que "la evaluación, con independencia del momento en que se realice, puede contemplar todas las etapas de la intervención (GV, n.d.:14) y que, por tanto, esto no supone un elemento de distorsión.

7 Ya que atiende a los componentes internos del programa y juzga la pertinencia del programa o coherencia entre sus distintos componentes.

8 La investigación está limitada al PCU y a su relación con dos de los programas del Plan de Investigación, y no al PDCD en su totalidad.
} 
objetivos para la coherencia interna; y matriz de valoración de la compatibilidad de la intervención con otros programas (programas de Investigación y Difusión de Cuestiones Estratégicas de Interés, y de Investigación Histórica) para la coherencia externa.

\section{Evaluación de diseño del Programa de Colaboración con las Universidades}

\subsection{Un contexto de recelos e intereses mutuos}

La política de fomento de la cultura de defensa comenzó a definirse en la década de los noventa. Su principal interés residía en que la sociedad conociese las líneas generales de la defensa nacional y dispusiera de mayor información sobre los compromisos de España en el marco de la seguridad internacional. El aislamiento internacional de España, junto con una Ciencia Política todavía emergente (Jerez Mir y Luque $)^{9}$, entre otros factores, provocaban que las materias relacionadas con los estudios militares, la defensa o la seguridad internacional se encontraran en un estado de investigación y análisis, en el mejor de los casos, incipiente. Además, los condicionantes históricos, por causas sociales, políticas y propiamente militares, eran motivo de un recelo, cuando no abierto rechazo, hacia "lo militar". De ahí que, en sus primeros antecedentes, como en la Directiva de Defensa Nacional I/1006, se afirmase la necesidad de "afianzar la sintonía entre la sociedad y sus Ejércitos sobre la base de un mayor conocimiento".

Todos estos condicionantes estaban presentes en las aulas y claustros universitarios. Las razones para este "desencuentro" se pueden encuadrar en cuatro variables clave (Bueno, 2016a): los motivos históricos ya apuntados, disonancias epistemológicas ${ }^{10}$, la cultura institucional castrense ${ }^{11}$ y el tardío interés en España por los estudios sobre seguridad y defensa, fruto en buena medida de ese aislamiento ya mencionado.

La iniciativa para tratar de revertir esta situación alcanzó rango de política pública de la defensa con la publicación en el año 2000 del Libro Blanco de la Defensa y de la Directiva de Defensa, documentos de carácter político-estratégico de primer nivel que la definieron de manera oficial como objetivo de la política de defensa española. De manera paralela, en el año 2001 se creó la Dirección General de Relaciones Institucionales (DIGERINS), dependiente de la Secretaría General de Política de Defensa (SEGENPOL). La DIGERINS era responsable de la planificación y desarrollo de la política cultural del Ministerio y de las relaciones institucionales con otros organismos del Estado e instituciones, como las propias universidades.

Dicha dirección general fue la encargada de confeccionar el PDCD como instrumento de planificación para ordenar y estructurar los objetivos, principios, órganos de dirección y coordinación, y ámbitos de actuación de cada uno de los planes deri-

9 Aunque la seguridad internacional y la defensa son campos de investigación multidisciplinares, especialmente en el mundo anglosajón se han enmarcado habitualmente dentro de la disciplina de la Ciencia Política.

10 Referida a aquellos sectores académicos que, desde posiciones ideológicas antimilitaristas, rechazaban las relaciones de las universidades con el ejército y hasta la legitimidad misma de la investigación en asuntos militares o estudios estratégicos.

11 Relacionada con actitudes refractarias por parte de los militares a que los civiles pudieran analizar temas vinculados con la esfera castrense debido a los valores particulares y distintos de dicha institución con respecto a la sociedad. 
vados. Por su parte, dichos planes establecen de manera concatenada los principios, objetivos, programas, acciones, etc., a desarrollar en cada sector designado. La DIGERINS, el Estado Mayor de la Defensa y los Cuarteles Generales de los Ejércitos y de la Armada fueron los encargados de elaborar los planes derivados. Entre ellos, el PCU, enmarcado en el PCSE.

La puesta en marcha de estos planes y programas quedó bajo la responsabilidad de varios organismos: integrados entonces en la DIGERINS, el Instituto Español de Estudios Estratégicos (IEEE) ${ }^{12}$, la Subdirección General de Patrimonio HistóricoArtístico y la Subdirección General de Relaciones Sociales y Comunicación de la Defensa; el Centro Superior de Estudios de la Defensa Nacional (CESEDEN); y, por último y en sus respectivos ámbitos de competencia, también el Estado Mayor y el Estado Mayor de los Ejércitos y de la Armada. Esto último implicaba a su vez que las diferentes unidades repartidas por todo el territorio serían las encargadas de participar o promover actividades vinculadas con la difusión de la cultura de defensa en su área geográfica.

Esa configuración ha permitido celebrar acuerdos de las respectivas universidades de referencia (atendiendo a criterios principalmente territoriales), no sólo con esos órganos centrales de la Defensa, sino también con academias militares, centros de formación o unidades de investigación. Esta circunstancia se debe a que el PCU consideró que la autonomía universitaria, así como los esfuerzos que, ya desde los años noventa, se venían realizando para promover este tipo de actuaciones en los centros universitarios favorecerían dicha colaboración. Por otro lado, también se entendió como un aliciente el que las universidades aceptaran estas relaciones por sus propios intereses; es decir, con objeto de no quedarse al margen de la consolidación disciplinar que disfrutaban los asuntos relativos a la seguridad internacional. Y es que estos estudios experimentaron una fuerte expansión tras el fin de la Guerra Fría (Buzan y Hansen, 2009).

\subsection{El PCU como instrumento de actuación pública: objetivos y acciones}

Respectos a los objetivos del PCU, éste no cuenta con una enumeración propia, sino que están subsumidos en los del PCSE. Dichos objetivos se orientan a la necesidad de generar conocimiento sobre las cuestiones relativas a la seguridad y la defensa, el compromiso con la paz internacional o con la pertenencia a organismos de cooperación securitaria, como la OTAN. Específicamente, se desea promover el estudio, debate e investigación entre los alumnos universitarios. De forma lógica, estos propósitos encajan con el objetivo del PDCD de potenciar tales actividades en cuestiones relacionadas con la paz, la defensa y la seguridad. Además, quiere formar expertos con conocimientos sobre seguridad y defensa que participen en el debate público. Una cuestión que no se circunscribe a las universidades, aunque, obviamente, sus académicos serían parte integrante de quienes contribuyesen al pensamiento estratégico español.

12 Posteriormente, en 2012, el IEEE se reincorporó a la estructura orgánica del CESEDEN, teniendo dependencia funcional de SEGENPOL en lo relativo a la difusión de la cultura de seguridad y defensa. Con este mecanismo, SEGENPOL es la responsable de firmar todos los convenios y subvenciones con las diferentes universidades, mientras el IEEE apoya estas acciones. De este modo, se han puesto en marcha congresos nacionales e internacionales, jornadas, cursos de verano, másteres, etc. 
Sobre las acciones concretas a implementar, se refiere principalmente a la consolidación y ampliación del volumen de seminarios, cursos de veranos, jornadas, etc., que se celebran, así como a aumentar el número de cátedras, títulos de posgrado o asignaturas en los grados. En líneas generales, se fija como forma de proceder el aportar ponentes militares o subvencionar parte de las actividades. Su foco de atención lo pone en los centros universitarios, de forma indiferente a su naturaleza pública o privada, o ubicación ${ }^{13}$.

Por otro lado, el PCU hay que vincularlo, tal y como se ha señalado, con el Plan de Investigación y sus programas derivados: 1) Programa de Sondeos y Estudios Sociológicos; 2) Programa de Investigación y Difusión de Cuestiones Estratégicas de Interés; y 3) Programa de Investigación Histórica. Los dos últimos se relacionan de manera más estrecha con el ámbito universitario y, por tanto, han sido analizados aquí para evaluar la coherencia externa del PCU ${ }^{14}$. Si bien, el Plan de Investigación es más amplio, pues dirige su atención también a think-tanks e instituciones homólogas con objeto de estimular el aumento del número de análisis y eventos sobre seguridad y defensa.

En los otros dos programas sí se subraya el papel que pueden desempeñar los centros universitarios en la promoción de temas de investigación sobre sus ámbitos de actuación. Estos programas, con estructura parecida al PCU, proponen una serie de acciones en línea similar ${ }^{15}$ : realización de jornadas y seminarios especializados de investigación, constitución de grupos de trabajo en el CESEDEN y en el IEEE para consolidar esas áreas de trabajo, celebración de cursos de verano o estímulo de tesis doctorales. Con estas acciones se pretende conseguir que la colaboración entre universidades y las FAS fomente la investigación en estas materias.

\subsection{Resultados de investigación sobre la racionalidad, pertinencia y coherencia interna y externa del programa}

\section{a) Racionalidad}

Para comprender mejor la racionalidad intrínseca del programa, se ha estudiado el diagnóstico de los problemas contemplados en el PDCD, ya que ni el PCSE ni el PCU dedican apartado alguno al respecto. En primer término, se analiza la propia

13 Sí cabe reseñar una mención expresa a su especial promoción en las facultades de Ciencias de la Información, bajo la premisa de que sus estudiantes, futuros comunicadores y periodistas, podrían ofrecer una mejor y más completa información a la opinión pública sobre estas materias. Con esta premisa se aprecia también el propósito específico que tiene esta política pública de influir en los estados de opinión pública, a mayor abundamiento con el Plan derivado de Comunicación Social, dirigido a estos profesionales y con fines similares.

14 El primer programa, el de Sondeos y Estudios Sociológicos, no es analizado en este artículo, al entenderse que los responsables del mismo decidieron plantearlo desde otro enfoque distinto al de la colaboración con las universidades. Por supuesto que sociólogos u otros académicos pueden contribuir significativamente a identificar los indicadores y motivaciones que expliquen el interés o no, el aprecio o desafección, por los temas de seguridad y defensa, y el ejército particularmente. Sin embargo, el programa se concentra en el trabajo de otras instituciones, como think-tanks (en un momento en el que había pocos centros de pensamiento de este tipo o se empezaban a crear; sin ánimo de exhaustividad, cabe destacar aquí el Grupo de Estudios Estratégicos, el Instituto de Cuestiones Internacionales y Política Exterior, y el Real Instituto Elcano de Estudios Internacionales y Estratégicos) o centros públicos de investigación (como el Centro de Investigaciones Sociológicas y su encuesta "La Defensa Nacional y las Fuerzas Armadas", encargada desde al año 1997 por el Ministerio de Defensa) y, en especial, se dirige hacia trabajos que ex profeso encargue el Ministerio.

15 No obstante, se señala que las actividades corresponderán al Plan derivado de Investigación siempre que no se hayan establecido previamente en el PCSE. 
Tabla 1. Calidad de la definición de los problemas.

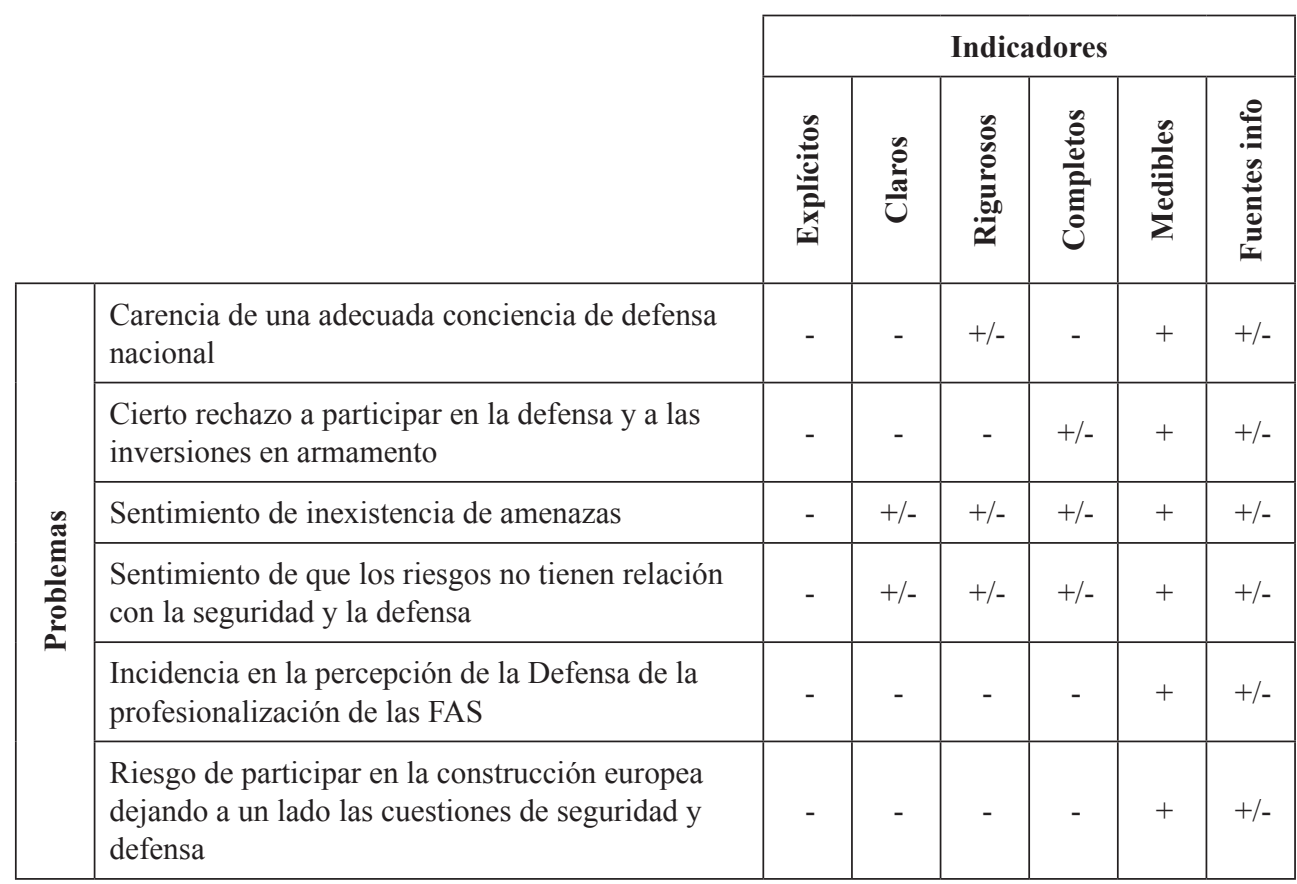

Elaboración propia. Los valores dados fijan tres posiciones: adecuada conceptualización del problema $(+)$, deficiente $(-)$, o correcta, pero susceptible de mejora (+/-).

a Significado de los indicadores: Explícitos: resaltados y no incluidos en una estructura discursiva. Claros: no presentan ambigüedades sobre su contenido. Rigurosos: proporcionan una "imagen fiel" de la población objetivo. Completos: caracterizan exhaustivamente la realidad del objeto. Medibles: deben aparecer cuantificados o, al menos, ser mesurables. Fuentes de información: se han de especificar las fuentes de información y trabajos sobre los trabajos de investigación realizados para el diagnóstico.

calidad de la definición de los problemas; en segundo, se examina el análisis de diagnóstico expuesto en el plan.

De la introducción del PDCD es posible deducir hasta seis problemas en los que se considera que hay que intervenir, recogidos en la Tabla 1. No obstante, cada uno admite diversas interpretaciones sobre su naturaleza y elementos configuradores, amén de que su mero encaje en la descripción narrativa dificulta completar su definición o la relación (si la hubiera) entre los mismos. Esta descripción poco nítida influye igualmente en su rigurosidad y en la población u objetos que se verían afectados, dado que sólo hay referencias genéricas a la sociedad o a la ciudadanía, no citándose específicamente a la comunidad universitaria. En la exposición tampoco se entra en conceptualizar el término de cultura de defensa, más allá de una escueta noción. El problema de la "escasa cultura de defensa" no se encuentra cuantificado, aunque bien sería posible su medición. Tampoco la dimensión relativa a las fuentes de información utilizadas es idónea, dado que hay una escasa concreción de las referencias empleadas ("análisis de pensadores y sociólogos, sondeos de opinión y análisis sociológicos") ${ }^{16}$.

16 Ciertos autores, como (Noya, 2013), afirman que el problema de la escasa cultura de defensa se amolda a un sesgo conservador en su conceptualización, debido a las posiciones ideológicas de algunos de sus primeros estudiosos. 
Figura 1. Diagrama causa-efecto del problema objeto de intervención pública.

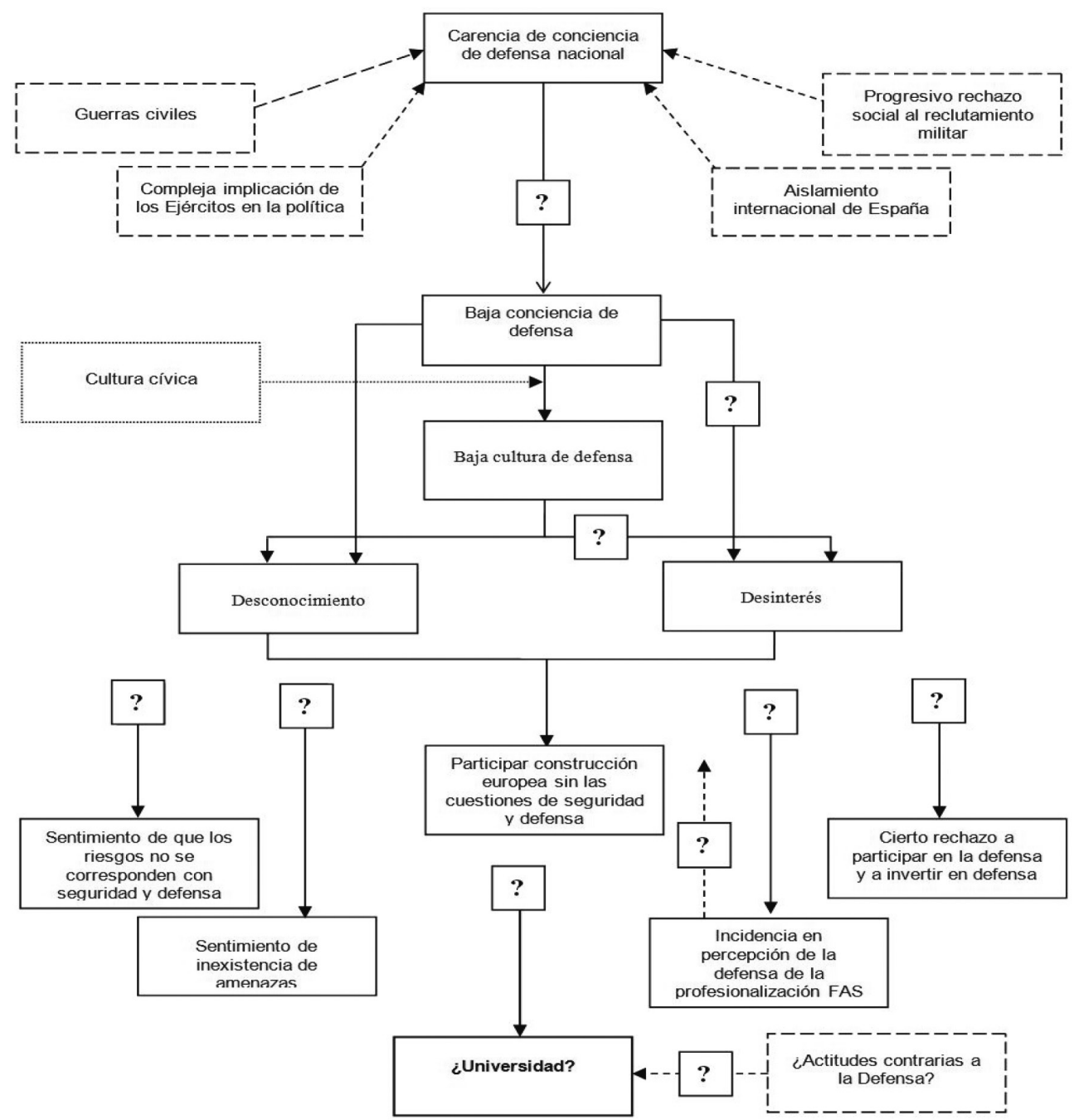

Elaboración propia. El diagrama causa efecto determina de forma descendente dicha relación. Los efectos se muestran en línea negrita, mientras que las causas lo hacen con línea discontinúa. Los signos de interrogación muestran momentos donde la relación causal no se explica o se hace de forma escasa. La "cultura cívica" aparece con un contorno punteado puesto que no queda claro si es causa o efecto del problema, si es un problema a resolver o un elemento para su solución.

Otra dimensión fundamental es el examen de la relación causa-efecto del problema al que trata de dar respuesta el plan y el programa, explicado en la Figura 1. El PDCD comprende cuatro causas de la "carencia de conciencia de defensa nacional", aunque deja entrever que puede haber más; esta falta de exhaustividad representa ya de por sí un error. El apartado introductorio menciona la falta de conciencia de defensa como efecto final de escasa cultura de defensa, que estaría a su vez determinada por el desinterés y el desconocimiento de la población sobre los asuntos de defensa y seguridad. No queda claro, por tanto, qué elementos corresponden a la conciencia y cuáles a la 
cultura $^{17}$, o si ambos están presentes en las dos dimensiones. La exposición concluye que este desinterés y desconocimiento pueden ser un obstáculo para la participación en la Unión Europea ${ }^{18}$. De esta manera, los demás problemas enumerados quedan desconectados de esta eventual escasez de cultura de defensa. Además, uno de ellos, la "incidencia en la profesionalización"19, es tremendamente ambiguo, pues no es sencillo discernir si es un problema de origen, como parece presentarse, o un efecto a considerar de todo ello.

Por último, cabe apuntar a la inexistencia de una mención expresa a la universidad o la eventual incidencia o no de tales déficits en dicho ámbito. Se observa, por tanto, que pese a tener un programa específico de colaboración y otros dos relacionados, este ámbito de actuación queda huérfano de referencia alguna. Esto podría causar, por ejemplo, que no se hubiesen contemplado problemas específicos para su implementación, como la presencia de actitudes refractarias o recelosas de la colaboración con los militares entre la comunidad universitaria.

\section{b) Pertinencia}

En cuanto a la pertinencia, potencial y formal, de los objetivos marcados por el programa, ésta se analiza a través de un análisis DAFO.

Del examen de la Tabla 2, destacan como fortalezas la claridad y concreción de los objetivos, pues reflejan adecuadamente el fin que persiguen y se corresponden con un único propósito. Otra fortaleza estriba en que varias de los objetivos ya venían desarrollándose mediante varias de las acciones propuestas en el programa, como la creación de cátedras universitarias o la celebración de cursos y jornadas sobre estas materias ${ }^{20}$.

Por otro lado, como oportunidades, los objetivos se adaptan a las particularidades locales de su ámbito de actuación o a cómo evolucionase la implantación del programa. Una oportunidad que se nutre del interés que estos asuntos iban suscitando en los centros universitarios. En este sentido, otro aliciente para las universidades podía ser la posibilidad de recibir subvenciones externas para la puesta en marcha de estas actividades.

Por el contrario, se manifiestan una serie de debilidades que podrían repercutir especialmente en la evaluación de los efectos de la intervención pública. Esta circunstancia está causada por cómo se formulan los objetivos, siempre enunciados mediante verbos "débiles" ("apoyar", "estimular", "fomentar" ...); para su medición, sería preferible que reflejasen acciones específicas orientadas a com-

17 La definición de los conceptos de cultura y conciencia de defensa vienen sufriendo de una confusión terminológica que puede dificultar su correcta comprensión (Bueno, 2016b) y, con esto, la formulación de alternativas para responder a la problemática planteada.

18 La puesta en marcha de esta política coincidía con un momento de mayor profundización en la unión política de la UE y el lanzamiento de diversas iniciativas de seguridad y defensa como los Battlegroups o el incremento de las misiones en el exterior.

19 Se refiere al fin del servicio militar obligatorio y al requerimiento de conformar unas FAS puramente profesionales, ya sin soldados de reemplazo. Por tanto, la preocupación era si habría recursos humanos suficientes para responder a las necesidades de la defensa, considerando que una parte significativa de la sociedad no tenía interés o sentía desafecto por el ejército y las cuestiones militares.

20 Durante la década de 1990 se pusieron en marcha diversas iniciativas enmarcadas en lo que posteriormente se conocería como promoción de la cultura de defensa, como la creación del Instituto Universitario General Gutiérrez Mellado o la constitución de diversas cátedras universitarias con el apoyo del CESEDEN. 
Tabla 2. Calidad de los objetivos.

\begin{tabular}{|c|c|c|}
\hline \multirow{6}{*}{$\begin{array}{l}\text { Análisis } \\
\text { Interno }\end{array}$} & Debilidades & Fortalezas \\
\hline & Formulación débil & Concreción \\
\hline & Complejidad en su medición: fácil medir & Claridad \\
\hline & productos, muy dificiles efectos & Responden a un solo fin \\
\hline & No fijan temporalización & Varios de ellos ya se encuentran en \\
\hline & No recogen cómo proceder & ejecución \\
\hline \multirow{5}{*}{$\begin{array}{l}\text { Análisis } \\
\text { Externo }\end{array}$} & Amenazas & Oportunidades \\
\hline & No contemplan la existencia de & Flexibilidad \\
\hline & corrientes o sectores refractarios & Carácter amplio \\
\hline & No especifican financiación & Fácil desarrollo \\
\hline & & Atractivo por interés del área \\
\hline
\end{tabular}

Elaboración propia.

portamientos mesurables "disminuir", "aumentar" ...). De aquí nace otra dificultad para conocer la efectividad del plan: la mera promoción de una actividad no garantiza un resultado. Ahondando en esta cuestión: incluso el incremento de jornadas, seminarios, cátedras, et., esto es, los productos de la intervención, no tiene por qué añadir información sobre sus efectos: quizá se esté actuando con individuos, docentes o estudiantes, que poseen ya una sensibilidad e interés por estas cuestiones. Igualmente, la medición de resultados se puede ver complicada por la falta de temporización en los objetivos. Posiblemente el carácter flexible que se quería imprimir al programa disuadiera de fijar una fecha, al menos tentativo, para su ejecución. No obstante, esto es una grave carencia en el largo plazo, en tanto que prolongar sine die su acción elude establecer un momento obligatorio que lo revise y evalúe, y, con ello, un momento para plantear la necesidad de una reforma, reformulación, etc. Por último, se observa la ausencia de indicaciones acerca de cómo proceder para la implementación de los objetivos. Se aprecia un enfoque incrementalista y de claro path dependency conforme a los criterios, actuaciones, y formatos de colaboración que ya se venían desarrollando. Por ende, surge la duda de cómo se implementarían, o bajo qué criterios y condiciones, aquellas otras iniciativas que no contasen con algún antecedente o referente previo.

En cuanto a las amenazas, la no dotación expresa de recursos (sólo una mención genérica en el PDCD a las partidas presupuestarias afectadas) ni una jerarquización o distribución clara de los organismos encargados ${ }^{21}$, puede ser causa de una infrafinanciación de actuaciones de mayor entidad, pero menos relevantes o el solapamien-

${ }^{21}$ Aunque el IEEE cumple un papel esencial, por encima de él se sitúan la DIGERINS y la SEGENPOL, ésta última responsable en la actualidad del Instituto. De manera horizontal, otros centros también se han de encargar de la promoción de la cultura de defensa en sus respectivas áreas geográficas o de competencias, como se ha señalado con anterioridad. 
to de recursos ${ }^{22}$. Otra amenaza para el buen funcionamiento de esta política pública viendo dado por las dificultades para acceder al mercado laboral de todos aquellos estudiantes que se especialicen en este campo (Jordán, 2016); una cuestión totalmente soslayada en el programa. Por último, y como ya se ha apuntado, aquí también se detecta la desatención hacia aquellos actores o sectores universitarios con actitudes contrarias a la defensa y el ejército. Por consiguiente, tampoco se plantean acciones o actuaciones para tratar de responder a esta problemática.

En conclusión, el análisis DAFO muestra que la calidad interna de los objetivos es adecuada en general. Si bien, también presentan elementos de crítica: en cuanto a la dificultad para evaluarlos y determinar sus efectos; respecto a las opciones profesionales que podría ofrecer esta formación académica, extracurricular o investigadora más allá de las propias FAS; y, por último, sobre las posibles resistencias que encontrara a su despliegue.

\section{c) Coherencia interna}

La coherencia interna estudia la planificación y estructura jerárquica de los objetivos previstos. En la Figura 2 se recogen tanto los objetivos generales del PDCD como específicos del PCSE, así como las acciones propuestas en el PCU.

El análisis de la coherencia interna parte de un problema ya detectado en el estudio de la calidad del problema, y es la ausencia de conceptualización acerca del problema de la escasa cultura de defensa en las universidades española. Si bien, a partir del análisis bibliográfico, es posible completar la valoración de la jerarquía de objetivos.

En el primer nivel de objetivos se observan algunos aspectos que requerirían de una revisión del diseño. El fomento del conocimiento sobre la paz, la seguridad internacional y la defensa, se correspondería con más de un objetivo del PDCD. Esta disonancia está provocada porque no queda claro si la promoción de esta política excluye o no a la universidad del ámbito educativo (toda vez que el sistema educativo general comprende la Educación Secundaria Obligatoria y Bachillerato-Formación Profesional). Se presenta explícitamente para uno (Objetivo b), pero queda la duda con respecto a los demás (Objetivos $e, g, h$ ), incluyendo la contribución a la conformación del pensamiento estratégico o a la de una comunidad de expertos.

Estos dos últimos resultan primordiales para el devenir favorable de la cultura de defensa. Sin embargo, no queda claro cómo se interrelacionan. Sólo una lectura atenta e integrada del PDCD, del PCSE y de sus respectivos programas permite entender mejor su configuración, lo que resiente la jerarquización y linealidad de los objetivos. Esta falta de vinculación jerárquica podría conllevar la inadecuada coordinación de esfuerzos, pues es lógico pensar que muchos de estos objetivos encuentran espacio comunes para su desarrollo ${ }^{23}$.

22 Cabe pensar, por ejemplo, que, ante el elevado número de universidades existentes en la Comunidad de Madrid, junto con la alta concentración de centros vinculados al Ministerio de Defensa, se produciría una aglutinación de actividades en esta región frente a una infrarrepresentación de otras zonas del Estado. Como consecuencia, objetos u escenarios quedarían desatendidos antes una eventual falta de coordinación y priorización de competencias y recursos.

23 Por ejemplo, si el PCU promueve la creación de cátedras, la realización de tesis doctorales o la celebración de cursos de verano, pareciera positivo que haya una mejor compenetración con esos otros programas y objetivos (el de Investigación y Difusión de Cuestiones Estratégicas de Interés, y el de Investigación Histórica) que igualmente impulsan seminarios de investigación o doctorados. 
Figura 2. Jerarquización de objetivos y acciones.

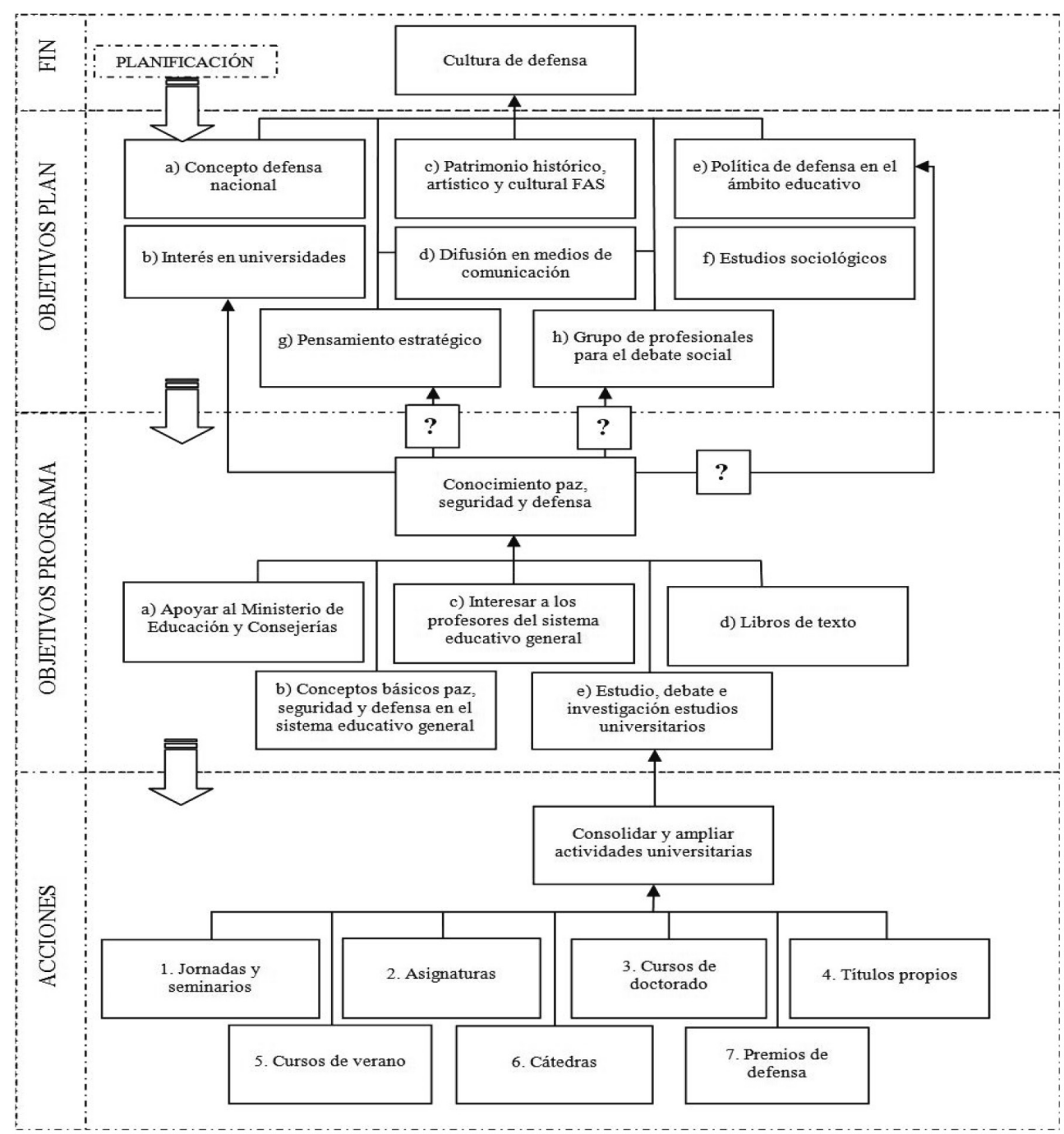

Elaboración propia. Las líneas flechadas establecen un vínculo, en este caso bottom-up, entre acciones y objetivos, y objetivo-objetivo, relación analizada en sentido contrario a cómo se produce la implementación.

\section{d) Coherencia externa}

La coherencia externa del programa compara sus objetivos con los de otros programas que guardan estrecha relación con él, bien por su aplicación en el mismo espacio físico, bien por si simultaneidad temporal. En este caso, se ha analizado la coherencia entre el PCU y los programas de Investigación y Difusión de Cuestiones Estratégicas de Interés (PIDCE), y de Investigación Histórica (PIH), comparación expuesta en la Tabla 3.

En línea con las conclusiones finales del anterior apartado, se advierte una clara superposición de objetivos entre los tres planes. Esto es así porque el PCU está enfo- 
Tabla 3. Coherencia externa del programa.

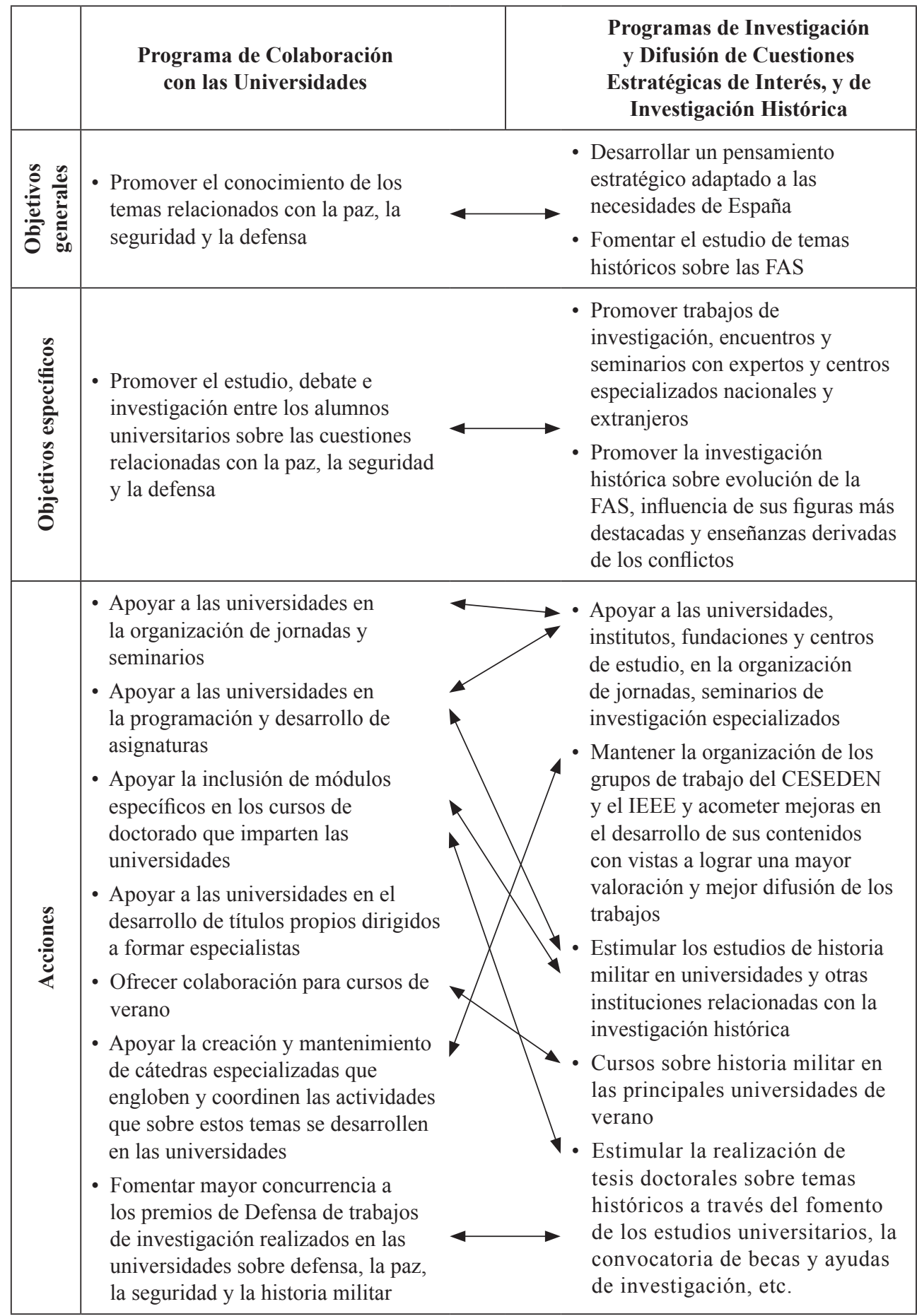

Elaboración propia. Las líneas flechadas indican la similitud o coincidencia en objetivos o acciones entre los programas. 
cado particularmente a la formación de estudiantes, mientras que el PIDCE y el PIH se dirigen al sector docente e investigador. Una circunstancia que no impide valorar la clara convergencia entre los mismos, tanto en sus objetivos generales como específicos. Por tanto, se podría pensar que las mayores diferencias vendrían entonces evidenciadas en sus respectivos niveles de actuaciones. No es así. Las acciones del PCU casan con buena parte de las acciones previstas para los otros programas. Todos comparten el fomento de seminarios, jornadas, cursos de verano, etc., al igual que la realización de tesis doctorales.

El único matiz lo aporta el PIH, el cual, se centra obviamente en su área de estudios. Los decisores de defensa quisieron dedicar especial atención a la historia bajo la argumentación de que el desconocimiento de la historia militar es una de las causas de la escasa cultura de defensa. Sin embargo, sorprende una priorización tan diferenciada, cuando otras disciplinas tienen igual interés por el estudio de las cuestiones relativas a la paz, seguridad internacional y defensa, y la multidisciplinariedad de las investigaciones es hoy en día una necesidad.

Por otra parte, es difícil entender la separación existente entre la creación de cátedras y la formación de especialistas, por un lado, con la participación de expertos del campo universitario en seminarios y grupos de trabajo de Defensa, por otro. Exponer de manera clara la complementariedad de ambas iniciativas ayudaría sin duda a su retroalimentación. Entender la superposición de objetivos y actuaciones podría contribuir a ahorrar esfuerzos y aunar sinergias, además de evitar la concentración de actuaciones en determinados espacios (vid. nota al pie 22).

\section{Conclusiones}

La investigación evaluativa de diseño del Programa de Colaboración las Universidades del Ministerio de Defensa ha valorado cuatro dimensiones de sus elementos configuradores: la racionalidad, la pertinencia, la coherencia interna y la coherencia externa. Tras el examen de su racionalidad y pertinencia, poniéndolo en relación con el PDCD y PCSE de los que deriva, se juzga de manera crítica la primera y positiva la segunda. Las dimensiones sobre la coherencia presentan ambivalencias en su juicio. A continuación, se detallan estas consideraciones.

El fin perseguido por esta política se puede deducir del texto del plan y del estudio de la bibliografía. El PDCD presenta una conceptualización poco enriquecida del problema a tratar: su naturaleza y características no son descritas con profusión, y son fácilmente interpretables desde distintos puntos de vista. Esto es algo que debería evitar cualquier documento de intervención pública. Asimismo, existe cierto desorden en el momento de establecer relaciones de causas y efectos. De esta circunstancia se desprende la posibilidad de que, en el transcurso de la implementación del programa, puedan surgir distintas formas de enfocar los proyectos de colaboración o que permanezcan sin cubrir aspectos del problema. Preocupa la nula referencia a la existencia de actitudes contrarias o renuentes a la relación con los militares y la defensa en las universidades.

Por el contrario, los objetivos de la política se encuentran en líneas generales adecuadamente identificados, probablemente por el consenso en torno a la meta a lograr, esto es, la promoción de la cultura de defensa. Éste es la dimensión 
juzgada como más positiva del programa. La principal debilidad del planteamiento de los objetivos es la dificultad de evaluar los efectos y resultados de la política. Para este fin, la formulación de objetivos debería ser concreta y mesurable. Igualmente, la inexistencia de límites temporales entorpece precisar un momento a partir del cual el plan o los programas hayan de ser revisados. Esto deja la puerta abierta a que el decisor no tenga presión alguna en este sentido y, por tanto, a que el programa de actuación se dilate en el tiempo sin saber si se están consiguiendo o no los resultados esperados, o si su implementación presenta desviaciones respecto al plan inicial. De hecho, ésa es la situación actual: tres lustros después de su publicación, todavía no se ha llevado a cabo una revisión del PDCD.

En cuanto a la coherencia interna y externa del programa, se obtienen dos conclusiones divergentes. Se puede juzgar la coherencia interna del programa como muy adecuada, ya que los objetivos y las acciones previstas explicitadas muestran una estructura lógica y perfectamente articulada. Además, no son apreciables contradicciones significativas entre los diferentes niveles, lo que refuerza esta valoración. No obstante, sí sería deseable una mayor claridad en cuanto a la conexión del objetivo del PCU y del PCSE, con la globalidad de los objetivos del PDCD, pues se advierte la interrelación de varios objetivos, lo que podría ocasionar distorsiones.

Respecto a la coherencia externa, se estima que la coordinación entre el PCU y los PIDCE y PIH, respectivamente, debería ser mucho mayor. La superposición de objetivos no se corresponde con una mayor cooperación entre iniciativas de acción; la obediencia a objetivos distintos no lo justifica. Esto provoca que no haya continuidad entre las distintas etapas de formación, especialización y profesionalización en las universidades, o que no se vincule el trabajo en el mundo académico con el debate social y estratégico acerca de las cuestiones relativas a la seguridad y la defensa nacional.

En conclusión, y en respuesta a si la alternativa planteada es la mejor posible para solventar las necesidades planteadas, se puede valorar que el planteamiento del plan y el programa es adecuado, puesto que el fomento y difusión de la cultura de defensa en las universidades españolas presentan unos objetivos que indican la dirección a seguir y, por esta razón, las acciones previstas son correctas. Sin embargo, posee varias deficiencias en su formulación y que deberían ser subsanados: 1) La conceptualización del problema no ahonda entre causas y efectos, lo que debilitaría el despliegue de la actuación pública; 2) Obvia la presencia de elementos refractarios en los centros universitarios, un factor que obstaculizar su implementación; 3) Adolece de una nítida dotación o priorización en la asignación de recursos, pudiendo provocar ineficiencias en su asignación; 4) No establece una temporización para desarrollarlo y, entonces, proceder a su evaluación; 5) No se coordina el trabajo entre los distintos agentes competentes.

\section{Recomendaciones de política}

En relación con las conclusiones alcanzadas, en particular con aquellos elementos críticos de su programación, y las restantes valoraciones obtenidas de la investigación evaluativa, se proponen ahora una serie de recomendaciones de política que, 
como ideas-fuerza, podrían ser de utilidad para los decisores públicos en el proceso de elaboración del nuevo Plan Director de Cultura de Defensa. Este propósito pretende servir indirectamente al progreso en torno a una mayor cultura evaluativa en la Administración Pública española y, en particular, en la Administración de Defensa, como parte del "continuo proceso de racionalización y objetivación" (Alvira, 2000: 231) en el que se hallan inmersas. Por tanto, se explicitan a continuación cinco recomendaciones de política vinculadas, por un lado, con los objetivos y acciones a implementar, y, por otro, con la formulación misma de la intervención pública de cara a su futura evaluación.

Por una parte, el despliegue de los objetivos se beneficiaría de una conceptualización más profunda de los conceptos de cultura de defensa y conciencia de defensa, sobre todo para determinar cuál es la relación entre los mismos, así como delimitar claramente cuáles son las causas de su eventual ausencia y cuáles los efectos. Entender la cultura de defensa como integrante de la cultura política y, por ende, la existencia de distintas subculturas (Bueno, 2016b), ayudará a especificar los sujetos de atención de la política.

En relación con la presencia de diferentes subculturas, podría ser positivo prestar atención a aquellos sectores de la comunidad universitaria más renuentes o contrarios a la presencia de las FAS en las universidades. Plantear acciones para reforzar el diálogo y la interacción con estos grupos puede ser una herramienta para acercar posiciones y, de tal modo, enriquecer el debate público. Si no, el riesgo de "convencer" a los ya convencidos es elevado.

En esta línea, es oportuno proponer innovaciones en algunas de las acciones ya implantadas, alejándose en la medida de lo posible del incrementalismo seguido. No porque éste sea un enfoque erróneo (antes, al contrario, ha permitido consolidar exitosamente algunas de las iniciativas que se pusieron en marcha ya en los años noventa), sino porque crea incertidumbre acerca de si se están obviando áreas de posible interés o metas futuras. En relación con este punto, se pueden considerar iniciativas dirigidas a incrementar el papel de los expertos en seguridad y defensa en el desarrollo de la política de defensa, mediante labores de asesoramiento, consultoría o investigación. Se activaría y se aprovecharía de este modo "la infraestructura de cultura de defensa" (Fojón, 2017) para la producción de pensamiento y análisis estratégicos. Además, la asistencia pareja de expertos civiles y militares en los foros profesionales, académicos o sociales proyectaría los trabajos compartidos y las sinergias investigadores y analíticas. Aprovechar el actual interés de las universidades por reforzar la transferencia de conocimiento hacia la sociedad puede coadyuvar a esta labor.

Por otra parte, conviene establecer mecanismos que favorezcan la evaluación del PDCD. Esta circunstancia obliga a implantar procesos de evaluación durante todo el período de vida útil del plan, partiendo desde la misma evaluación de diseño hasta una evaluación de resultados. La cuantificación del número de jornadas, congresos o seminarios realizados constituye, sin lugar a dudas, un indicador excelente de la marcha del plan y de la generación de productos de cultura de defensa; pero no es suficiente. Por ello se ha de considerar igualmente instrumentos cualitativos de medición. Para este propósito, resulta fundamental disponer de un marco temporal que fije su revisión y así atender a eventuales desviaciones o errores en su implementación. En este sentido, se podría emular el plazo de seis años fijado por el ciclo de planeamiento de la defensa (OM 60/2015) para su examen y revisión, con períodos bianuales de actualización de acciones y actividades. 


\section{Documentos}

- Directiva de Defensa Nacional 1/2000, de 1 de diciembre.

- Directiva de Defensa Nacional 1/2004, de 30 de diciembre.

- Directiva de Defensa Nacional, 1/2008, de 30 de diciembre.

- Directiva de Defensa Nacional 1/2012, de julio.

- Directiva 5/2002, de 11 de enero, por la que se aprueba el Plan Director de Cultura de Defensa.

- Directiva núm. 138, de 16 de septiembre de 2003, por la que se aprueban los Planes Derivados del Plan Director de Cultura de Defensa.

- Estrategia Española de Seguridad (2011). "Una responsabilidad de todos". Gobierno de España.

- Estrategia de Seguridad Nacional. (2013). "Un proyecto compartido". Gobierno de España.

- Estrategia de Seguridad Nacional. (2017). "Un proyecto compartido de todos y para todos". Gobierno de España.

- Ley Orgánica 5/2005, de 17 de noviembre, de la Defensa Nacional.

- Ley 36/2015, de 28 de septiembre, de Seguridad Nacional.

- Libro Blanco de la Defensa (2000). Ministerio de Defensa.

- Orden Ministerial (OM) 60/2015, de 3 de diciembre, por la que se regula el proceso de Planeamiento de la Defensa.

- Revisión Estratégica de la Defensa (2003). Ministerio de Defensa.

\section{Bibliografía}

AA.VV. (2014). XXI Curso Internacional de Defensa: Potenciar la cultura de seguridad, una necesidad para comprender la defensa nacional. Madrid: Ministerio de Defensa.

Agencia de Evaluación y Calidad (2010). Fundamentos de evaluación de políticas públicas. Madrid: Ministerio Política Territorial y Admón. Pública Disponible en http://www.aeval. es/export/sites/aeval/comun/pdf/evaluaciones/Fundamentos_de_evaluacion.pdf [Consultado, 5 de febrero de 2017]

Aguilar Idáñez, M J y Ander-Egg, E. (1994). Evaluación de servicios y programas sociales. Buenos Aires: Lumen.

Alemán, C y Trinidad, A. (2012). Evaluación de servicios sociales. Pamplona: Thompson Reuters.

Alvira, F. (2000). "Metodología de la evaluación: la lógica de la evaluación”, en Trinidad, A. (coord.). Evaluación y calidad en las organizaciones públicas. Madrid: Instituto Nacional de Administración Pública.

- (1991). Metodología de la evaluación de programas. Madrid: Centro de Investigaciones Sociológicas.

Amezcua Viedma, C y Jiménez Lara, A. (1996). Evaluación de programas sociales. Madrid: Díaz de Santos.

Anguera, $\mathrm{M}^{\mathrm{a}} \mathrm{T}$ y Moscoso, S. (2008). "Aproximación conceptual en evaluación de programas", en Anguera, M. T., Chacón, S y Blanco, A. (eds.). Evaluación de programas sociales y sanitarios, Madrid: Síntesis.

Ballart, X. (1992). ¿Cómo evaluar programas y servicios públicos? Aproximación sistemática y estudios de caso. Madrid: Ministerio Administraciones Públicas. 
Ballesteros, M. Á. y Fernández Vargas, V. (2014). "Cultura de defensa”, en ARBOR, vol. 190, $\mathrm{n}^{\mathrm{o}} 765, \mathrm{pp} .1-2$.

Briones, G. (1991). Evaluación de programas sociales. México D.F.: Trillas.

Bueno, Alberto (2017). Los estudios de seguridad internacional y defensa en la Ciencia Política española: Análisis de las contribuciones académicas. Comunicación presentada en el XIII Congreso de la Asociación Española de Ciencia Política y de la Administración, Santiago de Compostela, 20-22 de septiembre.

- (2016a), "La política pública de fomento de la cultura de defensa en las universidades españolas: De su definición a su planificación", en Revista Ensayos Militares, vol. 2, n 2, pp. 31-48.

- (2016b), "La cultura de seguridad y defensa: una propuesta de análisis conceptual desde la ciencia política", en Revista del Instituto Español de Estudios Estratégicos, No. 8, pp. 41-70.

Buzan, B y Hansen, L. (2009). The evolution of international security studies. Cambridge University Press.

Cirera, A y Vélez, C. (2000). Guía para la evaluación de políticas públicas. Instituto de Desarrollo Regional.

Chelimsky, E. (1987). "Linking Program Evaluation to User Needs", en Palumbo, D. J. (ed.). The Politics of Program Evaluation. USA: SAGE Publications.

Colom, G. (2011). "El proceso de transformación militar en España (2004-2011), UNISCI, (27). Disponible en https:/www.ucm.es/data/cont/media/www/pag72497/UNISCI\%20DP\%2027\%20-\%20COLOM.pdf [Consultado, el 12 de julio de $2017]$.

Fernández-Ballesteros, R. (1996). Evaluación de programas. Una guía práctica en ámbitos sociales, educativos y de salud. Madrid: Síntesis.

Fernández Vargas, V y Rodríguez-Toubes, J. (2008). "La cultura de la defensa nacional en España", en $A R B O R$, vol. 184, n 2, pp. 1-2.

Fojón, E. (2017), "El gasto de defensa en España: objetivos estratégicos, militares e industriales", en ARI 44/2017, Real Instituto Elcano. Disponible en: http://www.realinstitutoelcano.org/wps/portal/rielcano_es/contenido?WCM_GLOBAL_CONTEXT=/elcano/elcano_es/zonas_es/ari44-2017-fojonlagoa-gasto-defensa-espana-objetivos-estrategicos-militares-industriales [Consultado, el 5 de junio de 2017]

García Palomero, I. (2014). "Estrategias de investigación, estudio y desarrollo aplicables en el fomento e innovación de la cultura de seguridad y defensa nacional", Instituto Español de Estudios Estratégico, Documento de Análisis 21/2013. Disponible en:http://www.ieee. es/Galerias/fichero/docs_analisis/2013/DIEEEA212013_Estrategias_de_Estudio_IGP. pdf [Consultado, el 31de marzo de 2017].

Gobierno Vasco (n.d.), Guía de evaluación de políticas públicas del Gobierno vasco. Disponible en: http://www.euskadi.eus/contenidos/informacion/evaluacion_coordinacion/es def/adjuntos/guia_evaluacion_gv_pip.pdf [Consultado, el 7 de abril de 2017].

Hogwood, B. W. y Gunn, L. A. (1984). Policy Analysis for the Real World. Oxford: Oxford University Press.

IEEE. (2015). "Cultura de seguridad y defensa: fundamentos y perspectiva de mejora”, en Cuaderno de Estrategia, no 172 . Madrid: Ministerio de Defensa.

- (2011), "La cultura de seguridad y defensa: un proyecto en marcha", en Cuaderno de Estrategia, $\mathrm{n}^{\circ} 155$. Madrid: Ministerio de Defensa.

Mir, M y Luque, J. (2016). “Treinta años de Ciencia Política en España: profesionalización, expansión y ajuste”, en Revista Española de Ciencia Política, nº 40, pp. 179-215. 
Jordán, J. (2017). “Una aproximación a la agenda institucional de la Comisión de Defensa del Congreso de los Diputados español”, en UNISCI, n 44. Disponible en http://www.ugr. es/ jjordan/comision-defensa.pdf [Consultado, el 2 de octubre de 2017].

- (2016). «¿Qué salidas profesionales tienen los estudios sobre Seguridad Internacional y Defensa en España?", en Grupo de Estudios en Seguridad Internacional, blog MOSAICO. Disponible en http://www.seguridadinternacional.es/?q=es/content/¿qué-salidas -profesionales-tienen-los-estudios-sobre-seguridad-internacional-y-defensa-en-0 [Consultado, el 10 de septiembre de 2017].

Lasswell, H. D. (1970). "The emerging conception of the policy sciences", en Policy Sciences, $\mathrm{n}^{\mathrm{o}} 12$, pp. 3-14.

López Mora, F y Ballesteros, M. Á. (2011). Ensayos sobre la cultura de defensa y la paz en la España actual. Córdoba-Madrid: UCO-DIGERINS.

Marrero, I. (2007). Hacia una nueva cultura de la defensa en España. Fundación Alternativas, Documento de Trabajo 11/2007. Disponible en http:/www.fundacionalternativas. org/observatorio-de-politica-exterior-opex/documentos/documentos-de-trabajo/hacia-una-nueva-cultura-de-la-defensa-en-espana [Consultado, el 6 de marzo de 2017].

Mèny, Y y Thoening, J. C. (1992). Las políticas públicas. Barcelona: Ariel.

Ministerio de Defensa. (2016). “Comparecencia de la Ministra de Defensa, María Dolores de Cospedal, ante la comisión de defensa del Congreso de los Diputados", 20 de diciembre. Disponible en: http://www.defensa.gob.es/Galerias/gabinete/ficheros_docs/2017/lineasgenerales-defensa-1.pdf [Consultado, el 5 de febrero de 2017].

Nirenberg, O. (2013). Formulación y evaluación de intervenciones sociales. Buenos Aires: Noveduc.

Noya, F. J. (2012). Visiones en el exterior: los españoles ante un mundo en cambio. Madrid: Tecnos.

Pérez Sánchez, M. (2005). “El estudio de las políticas públicas”, en Pérez Sánchez, M. (ed.). Análisis de políticas públicas. Granada: Universidad de Granada.

Pérez-Llantada, Ma C y López de la Llave, A. (1999). Evaluación de programas de salud y servicios sociales: metodología y ejemplos. Madrid: Dykinson.

Rossi, P. H. y Freeman, H. E. (1989). Evaluación. Un enfoque sistemático para programas sociales. México D.F: Trillas.

Rutman, L. (1984a). "Evaluability assessment", en Rutman, L. (ed.). Evaluation research methods: A basic guide. USA: SAGE Publications.

- (1984a), "Introduction", en Rutman, L. (ed.), Evaluation research methods: A basic guide. USA: SAGE Publications.

Stufflebeam, D. L. y Shinkfield, A. J. (1985). Evaluación sistemática. Guía teórica y práctica. Barcelona: Paidós y Ministerio de Educación y Ciencia.

Trinidad, A. (1995). La evaluación de instituciones educativas. El análisis de la Facultad de Ciencias Políticas y Sociología de la Universidad de Granada. Granada: Universidad de Granada.

Trujillo, H y García Sistac, J. A. (2006). Universidad de Granada-MADOC. Una colaboración proyectada hacia el futuro. Granada: Universidad de Granada.

Weiss, C. H. (1972). Evaluation research. New Jersey: Prentice-Hall. 
